Ciência Florestal, Santa Maria, v. 28, n. 1, p. 263-273, mar., 2018

ISSN 1980-5098@ @() DOI: http://dx.doi.org/10.5902/1980509831581

\title{
EFEITO DA SAZONALIDADE SOBRE O RENDIMENTO DO ÓLEO ESSENCIAL DE Piper gaudichaudianum KUNTH
}

\author{
EFFECT OF SEASONALITY ON THE ESSENTIAL OIL YIELD OF Piper gaudichaudianum KUNTH
}

\author{
Bianca Schindler ${ }^{1}$ Daniela Thomas da Silva ${ }^{2}$ Berta Maria Heinzmann ${ }^{3}$
}

\begin{abstract}
RESUMO
Piper gaudichaudianum Kunth (Piperaceae) é uma planta com ampla distribuição no território brasileiro. Além de sua importância ecológica, é utilizada na medicina popular e muitas de suas atividades biológicas já foram comprovadas. O objetivo deste trabalho foi verificar o efeito da sazonalidade sobre o teor de óleo essencial (OE) em folhas de Piper gaudichaudianum e averiguar a possível influência do processo de secagem do material vegetal no rendimento deste extrativo. Foram coletadas aleatoriamente folhas de indivíduos de uma população nativa de Santa Maria - RS, Brasil, ao longo de um ano. O OE foi extraído de folhas frescas e secas à temperatura ambiente pelo método de hidrodestilação. Quando verificada a presença de órgãos reprodutivos, os mesmos foram extraídos, a fim de examinar a sua influência no rendimento do OE de folhas. Para determinação do teor de umidade (TU\%), $20 \mathrm{~g}$ de folhas e $10 \mathrm{~g}$ de órgãos reprodutivos foram secos separadamente em estufa a $50^{\circ} \mathrm{C}$ até peso constante. O rendimento de $\mathrm{OE}$ de folhas $(\% \mathrm{~m} / \mathrm{m})$ foi calculado para o material fresco com base na massa seca, considerando o TU\%, bem como para o material seco à temperatura ambiente. Apenas pequenas variações sazonais nos teores de $\mathrm{OE}$ foram detectadas para as amostras analisadas, não havendo diferença estatística entre eles. O maior rendimento foi observado na primavera, tanto para o $\mathrm{OE}$ de folhas $(1,61 \pm 0,09 \%)$, quanto de órgãos reprodutivos $(5,17 \%)$, enquanto o menor teor de $\mathrm{OE}$ em folhas ocorreu no verão $(1,32 \pm 0,14 \%)$, a única estação em que não houve a presença de órgãos reprodutivos. A ausência de diferença significativa entre os rendimentos indica que não houve variabilidade sazonal quantitativa na produção de $\mathrm{OE}$ em folhas desta população. Portanto, a coleta deste órgão vegetal pode ser realizada em qualquer época do ano visando à obtenção deste extrativo. Uma vez que não foi verificada diminuição do teor de $\mathrm{OE}$ durante o processo de secagem à temperatura ambiente, esse processamento pós-colheita poderá ser adotado.
\end{abstract}

Palavras-chave: pariparoba; produto florestal não madeireiro; produção de óleo essencial; processamento pós-colheita.

\section{ABSTRACT}

Piper gaudichaudianum Kunth (Piperaceae) is widely distributed throughout Brazil. In addition to its ecological importance, it is used in folk medicine and a great number of its biological activities have been already proved. Thus, the aim of this research was to investigate the effect of seasonality on the content of essential oil (EO) in leaves of Piper gaudichaudianum and ascertain the possible influence of the drying process of the plant material in the yield of this extractive. Leaves were randomly collected from individuals of a native population in Santa Maria, RS state, Brazil, along a year. The EO was extracted from fresh and dried leaves by hydrodistillation. When reproductive organs were observed, they were extracted

1 Engenheira Florestal, Mestre pelo Programa de Pós-Graduação em Engenharia Florestal, Centro de Ciências Rurais, Universidade Federal de Santa Maria, Av. Roraima, 1000, CEP 97105-900, Santa Maria (RS), Brasil. bia. schindler@gmail.com

2 Engenheira Florestal, MSc., Doutoranda do Programa de Pós-Graduação em Engenharia Florestal, Centro de Ciências Rurais, Universidade Federal de Santa Maria, Av. Roraima, 1000, CEP 97105-900, Santa Maria (RS), Brasil.dthomasdasilva@gmail.com

3 Farmacêutica, Dr ${ }^{\mathrm{a}}$., Professora Associada do Departamento de Farmácia Industrial, Centro de Ciências Rurais, Universidade Federal de Santa Maria, Av. Roraima, 1000, CEP 97105-900, Santa Maria (RS), Brasil. berta. heinzmann@gmail.com

Recebido para publicação em 15/12/2014 e aceito em 19/08/2016

Ci. Fl., v. 28, n. 1, jan.- mar., 2018 
in order to examine their influence on the yield of leaf EO. To determine the moisture content (MC\%), 20 $\mathrm{g}$ of leaves and $10 \mathrm{~g}$ of reproductive organs were dried separately in an oven at $50^{\circ} \mathrm{C}$ until constant weight. The yield of leaf EO $(\% \mathrm{w} / \mathrm{w})$ was calculated for the fresh material based on the dry weight considering the $\mathrm{MC} \%$, and for the dried material at room temperature. Only small seasonal variations in EO content was detected for the analyzed samples, with no statistical difference between them. The highest yield was observed in the spring for EO from the leaves $(1.61 \pm 0.09 \%)$, and from the reproductive organs $(5.17 \%)$, while the lowest yields of leaf EO occurred in the summer $(1.32 \pm 0.14 \%)$, the only season which showed no presence of the reproductive organs. The absence of significant difference between the yields indicates no quantitative seasonal variability in production of EO in leaves of this population. However, yields did not differ statistically, indicating no seasonal variability of the EO content of the leaves of this population. Therefore, the collection of this plant organ can be performed at any time of the year in order to obtain this extractive. Since there was no decrease in the content of EO during the drying process at room temperature, this post-harvest processing can be adopted.

Keywords: pariparoba; non-timber forest products; essential oil production; post-harvest processing.

\section{INTRODUÇÃO}

Os produtos florestais não madeireiros (PFNM) são utilizados na alimentação, produção de medicamentos, cosméticos, construção de moradias, entre outros, sendo fundamentais para a subsistência de muitas comunidades. De acordo com a FAO (Organização das Nações Unidas para Agricultura e Alimentação), cerca de $80 \%$ da população de países em desenvolvimento usam os PFNM para suprir algumas de suas necessidades vitais (MACHADO, 2008). Adicionalmente, constituem uma oportunidade real para incrementar a renda familiar dos extrativistas (FIEDLER; SOARES; SILVA, 2008), além de tornar a floresta rentável e consequentemente valorizando-a (MACHADO, 2008). Entre os PFNM que vêm recebendo grande destaque encontram-se os óleos essenciais (OE) (SANTOS et al., 2003), cujo mercado internacional é estimado em 31,9 bilhões de dólares para o ano de 2014 (GOVINDASAMY; ARUMUGAM; SIMON, 2013). No entanto, a participação brasileira ainda é baixa e resulta especialmente da comercialização de $\mathrm{OE}$ de frutos cítricos, que são subprodutos da indústria de sucos de laranja, lima e bergamota (BIASI; DESCHAMPS, 2009; SPEZIALI, 2012).

Piper gaudichaudianum Kunth (Piperaceae), espécie conhecida popularmente por pariparoba ou jaborandi, é encontrada nas regiões Norte, Nordeste, Centro-Oeste, Sudeste e Sul do Brasil, Paraguai e Argentina (GUIMARÃES; VALENTE, 2001; GUIMARÃES et al., 2014). A importância do estudo dessa espécie reside em sua rusticidade, o que indica bom potencial para projetos de restauração ambiental, propiciando cobertura arbustiva em solos pobres (GUIMARÃES; VALENTE, 2001). O uso da espécie é muito diverso, as folhas frescas em infusão são utilizadas na medicina popular como analgésicas, enquanto as raízes frescas são usadas como anti-inflamatórias e nos casos de doenças do fígado (DI STASI; HIRUMA LIMA, 2002). As atividades biológicas descritas incluem: fungicida, larvicida, anti-inflamatória e analgésica (MOREIRA et al., 2001; DI STASI; HIRUMA-LIMA, 2002; LAGO et al., 2004; MORAIS et al., 2007). Além do mais, o $\mathrm{OE}$ de inflorescências (espigas) possui uma importante função ecológica na atração de morcegos da família Phyllostomidae (MIKICH et al., 2003).

Muitos fatores exercem influência tanto no rendimento, quanto na variabilidade da composição química dos $\mathrm{OE}$, como fatores ambientais, edáficos e climáticos, poluição atmosférica, bem como aqueles inerentes à própria planta, como ciclo vegetativo, idade e órgão vegetal. Sendo assim, fica evidente a necessidade do desenvolvimento de estudos que determinem as condições e épocas mais propícias para coleta de material vegetal, conduzindo a uma máxima produção e qualidade do extrativo (LIMA; KAPLAN; CRUZ, 2003; GOBBO-NETO; LOPES, 2007; FIGUEIREDO et al., 2008).

Neste contexto, o presente estudo teve como objetivo analisar o efeito da sazonalidade sobre o rendimento do OE de folhas de Piper gaudichaudianum coletadas em uma população localizada em Santa Maria - RS, Brasil, buscando definir a melhor época de colheita do material vegetal em função da maior produção de OE. Além disso, determinar a influência do processo de secagem sobre os teores de OE, a fim de averiguar a possibilidade do uso deste processo pós-colheita. 


\section{MATERIAL E MÉTODOS}

\section{Material vegetal}

A coleta de folhas de Piper gaudichaudianum foi realizada em uma população nativa, de indivíduos situados no sub-bosque de um remanescente florestal, em local úmido e com pouca luminosidade, localizado no município de Santa Maria, Rio Grande do Sul, nas imediações da BR-158, Km 318, sob as coordenadas $29^{\circ} 40^{\prime} 11.3$ " S e 53॰46' 15.8" O, com altitude em torno de $229 \mathrm{~m}$. As coletas foram realizadas no período da manhã, entre $8 \mathrm{~h} 30 \mathrm{~min}$ e $9 \mathrm{~h} 30 \mathrm{~min}$. A espécie foi identificada por Daniele Ferreira Monteiro e as exsicatas foram depositadas no Herbário do Departamento de Ciências Florestais (HDCF) na Universidade Federal de Santa Maria (UFSM) e uma duplicata no Herbário do Jardim Botânico do Rio de Janeiro (RB), sob registros 6.514 e 584.729, respectivamente. Para a avaliação da influência sazonal sobre o rendimento do $\mathrm{OE}$, foram coletadas folhas de forma aleatória, de indivíduos de toda a população da área em estudo, duas vezes em cada estação, no período de janeiro a dezembro de 2013 (Tabela 1). Nas estações em que houve a presença de órgãos reprodutivos (drupas ou espigas), os mesmos também foram coletados para posterior extração. O OE de órgãos reprodutivos foi extraído a fim de verificar a possível influência de sua presença sobre o rendimento do OE de folhas, uma vez que foi descrita a ocorrência de realocação de reservas na dependência do período vegetativo da planta (AMARAL et al., 2014). O termo utilizado em morfologia vegetal para a inflorescência desta espécie é espiga, uma vez que se refere às flores sésseis (sem pedicelo) (GONÇALVES; LORENZI, 2011), enquanto para o fruto é drupa (GUIMARÃES; VALENTE, 2001).

TABELA 1: Estações, datas das coletas e órgãos vegetais amostrados de Piper gaudichaudianum Kunth.

TABLE 1: Seasons, dates of collection and sampled vegetal organs of Piper gaudichaudianum Kunth.

\begin{tabular}{ccccc}
\hline Estações & Início e fim da estação & Data da coleta 1 & Data da coleta 2 & Órgão vegetal coletado \\
\hline Verão & $21 / 12-20 / 03$ & $14 / 01 / 2013$ & $07 / 03 / 2013$ & Folhas \\
Outono & $21 / 03-20 / 06$ & $08 / 04 / 2013$ & $10 / 06 / 2013$ & Folhas e inflorescências \\
Inverno & $21 / 06-20 / 09$ & $17 / 07 / 2013$ & $06 / 09 / 2013$ & Folhas e inflorescências \\
Primavera & $21 / 09-20 / 12$ & $03 / 10 / 2013$ & $04 / 12 / 2013$ & Folhas e frutos \\
\hline
\end{tabular}

\section{Caracterização do local de coleta}

O solo de Santa Maria está assentado sobre litologias das Formações Santa Maria, Caturrita, Botucatu e Serra Geral (MACIEL FILHO, 1990). Segundo informações da literatura, o solo da área de coleta é do tipo Neossolo Litólico (STRECK et al., 2008).

O clima é classificado como mesotérmico e úmido e, conforme a classificação de Köppen, é definido como tipo fundamental $\mathrm{Cfa}$, caracterizado como subtropical úmido com verões quentes, sem estação seca definida. A temperatura média anual é de $19,1^{\circ} \mathrm{C}$ e a precipitação pluviométrica mensal oscila entre 120,1 e 126,8 mm ao mês. A região está sujeita a geadas fracas e moderadas, o inverno é ameno, mas sujeito a ondas de frio provocadas pelo deslocamento frequente de anticiclones polares migratórios. A frequência média normal de geadas é de nove dias por ano, entre os meses de abril a outubro (HELDWEIN; BURIOL; STRECK, 2009).

Para o município de Santa Maria, a vegetação é classificada como Floresta Estacional Decidual (IBGE, 1992). No local de coleta, de acordo com a nova proposta de Oliveira-Filho (2009), é classificada como Floresta Latifoliada Estacional Rupícola. 


\section{Determinação do teor de umidade (TU\%)}

Para a determinação do teor de umidade, após cada coleta foram separadas aproximadamente $20 \mathrm{~g}$ de folhas frescas para secagem em estufa a $50^{\circ} \mathrm{C}$, até peso constante (aproximadamente 15 dias). $\mathrm{O}$ teor de umidade foi calculado através da equação 1 :

$$
T U \%=\frac{\text { massa úmida-massa seca }}{\text { massa úmida }} \times 100
$$

A determinação do TU\% foi utilizada nos cálculos de rendimento de OE, mais especificamente os valores de massa das folhas frescas em relação à base úmida (MF BU) e à base seca (MF BS) do material vegetal. A massa foliar à base seca (MF BS) foi corrigida através da equação 2:

$$
M F B S=\frac{(100-\mathrm{TU}) \times \mathrm{MF} \text { BU }}{100}
$$

Em que: MF BU é a massa das folhas frescas usadas nas extrações de OE.

Esta metodologia também foi aplicada às espigas, no entanto, com redução da quantidade amostrada (10 g). Neste caso, a nomenclatura foi adaptada para: massa de órgãos reprodutivos frescos em relação à base úmida (MOR BU) e à base seca (MOR BS).

\section{Extração do óleo essencial (OE)}

Folhas e órgãos reprodutivos frescos, bem como folhas secas por 15 dias à temperatura ambiente e ao abrigo da luz, foram fragmentados e submetidos separadamente à hidrodestilação para a extração do OE, em aparelho tipo Clevenger modificado, durante 3 horas. Neste método, o óleo destilado é retido em um tubo de vidro e a fase aquosa retorna automaticamente para o balão de destilação, sendo reutilizada (SARTOR, 2009). A extração de folhas foi realizada em triplicata, enquanto no caso de órgãos reprodutivos, devido à pequena quantidade de material disponível, foi realizada apenas uma extração a cada coleta. Como o OE se misturou ao hidrolato, este foi submetido à extração líquido-líquido em funil de separação, utilizando como solvente hexano previamente destilado (SILVA et al., 2009). Após a secagem da fração hexânica com sulfato de sódio anidro $\left(\mathrm{Na}_{2} \mathrm{SO}_{4}\right)$, o solvente foi evaporado em rotaevaporador a $30^{\circ} \mathrm{C}$, para obtenção do OE. O teor de $\mathrm{OE}(\% \mathrm{~m} / \mathrm{m})$ de folhas foi obtido a partir da massa de $\mathrm{OE}$, determinada em balança analítica, em relação à massa foliar à base úmida (MF BU), massa foliar à base seca (MF BS) e massa foliar seca à temperatura ambiente (FS). Cálculo equivalente foi aplicado ao OE de órgãos reprodutivos, que foi obtido apenas de material fresco, fornecendo o rendimento em relação à base úmida (MOR BU) e à base seca (MOR BS). A densidade ( $\mathrm{g} / \mathrm{mL}$ ) do $\mathrm{OE}$ (DOE) foi calculada através da equação 3:

$$
D O E=\frac{\text { massa de } O E(g)}{\text { volume de } O E(m L)}
$$

(Equação 3)

Os OE foram armazenados em frascos de vidro âmbar, vedados e conservados a $-4^{\circ} \mathrm{C}$.

\section{Análise estatística}

Os dados de rendimento de MF BU, MF BS e FS, não atenderam aos pressupostos de normalidade e homogeneidade de variâncias. Portanto, os dados de rendimento de primavera e verão foram transformados para logaritmo neperiano $(l n)$. Como no caso do outono e inverno não foi possível a transformação, os dados foram submetidos à estatística não paramétrica. Para verificação da normalidade e homogeneidade de variâncias, os dados foram submetidos aos testes de Shapiro-Wilk e Levene, respectivamente. Os dados de rendimento de MF BU, MF BS e FS foram comparados dentro da estação e entre estações pela análise de variância (ANOVA) de uma via, com os testes Tukey, Mann-Whitney e teste- $t$, que são apresentados pela 
média \pm erro padrão da média (EPM). As análises foram realizadas no software SigmaPlot, versão 11.0, $\operatorname{com} p<0,05$. Para a definição de grupos, os dados de rendimento obtidos para os OE (quatro estações, duas coletas por estação, extração em triplicata, perfazendo um total de $n=24$ para MF BU, MF BS e FS) foram submetidos à Análise Hierárquica de Cluster (AHC), com distância Euclidiana como medida de dissimilaridade e o método de ligação pela média UPGMA (GOTELLI; ELLISON, 2011) e obtendo o coeficiente de correlação cofenética ( $\mathrm{ccc}$ ) que indica que quanto mais próximo de $(1,0)$ o método de agrupamento foi adequado (ROHLF, 1970), com o auxílio do software R, versão 2.15.2, com o pacote vegan (OKSANEN et al., 2013).

\section{RESULTADOS E DISCUSSÃO}

O rendimento médio obtido para o OE de folhas frescas (MF BS) de Piper gaudichaudianum em um ano de coleta foi igual a $1,44 \pm 0,06 \%$, enquanto para folhas secas (FS) o rendimento médio anual correspondente foi de $1,31 \pm 0,06 \%$. O OE extraído de folhas frescas (MF BS) não apresentou variabilidade sazonal significativa em relação ao rendimento $(p<0,05)$. Os dados obtidos para os rendimentos de MF BU diferem estatisticamente de MF BS e FS, em cada período sazonal (Figura 1). A média da densidade do OE de folhas frescas (MF BU) e FS foi de aproximadamente $1,08 \mathrm{~g} / \mathrm{mL}$ para ambas as amostras, como apresentado na Tabela 2. Este dado sugere que a composição química das FS possivelmente não sofreu alterações durante o processo de secagem. O dado de densidade do OE é relevante, pois é próximo ao da densidade da água $\left(1 \mathrm{~g} / \mathrm{cm}^{3}\right)$, o que dificulta a separação do $\mathrm{OE}$ e da água em duas fases durante o processo de extração. Por outro lado, a elevada densidade também pode ser uma vantagem, por exemplo, quando é necessário homogeneizar amostras de $\mathrm{OE}$ em meios aquosos como o ágar, no caso da avaliação do seu potencial antifúngico (BURT, 2004; SOIDROU et al., 2013).

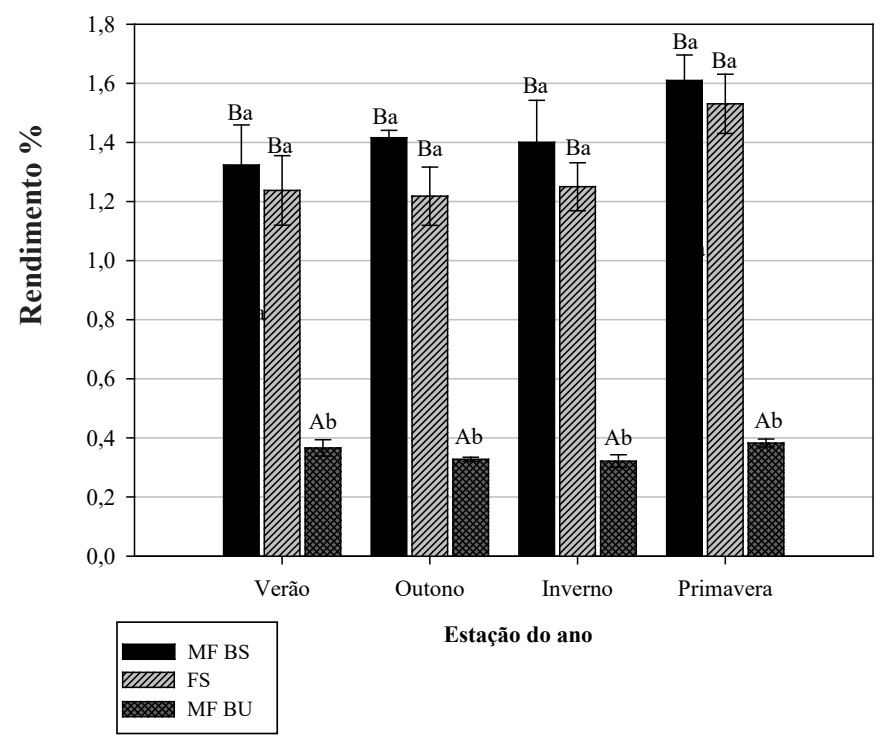

FIGURA 1: Rendimentos médios (\%) e Erro Padrão da Média dos óleos essenciais de folhas frescas à base seca (MF BS), à base úmida (MF BU) e de folhas secas (FS) de Piper gaudichaudianum Kunth, nas quatro estações do ano de 2013 (duas coletas por estação), em triplicata. Letras maiúsculas diferentes indicam diferenças significativas de rendimento entre as estações e letras minúsculas diferentes indicam diferenças significativas entre os rendimentos dentro da estação, pelos testes de Tukey, Mann-Whitney e teste-t, $p<0,05$.

FIGURE 1: Average yields (\%) and Standard Error Mean of the essential oils from fresh leaves based on dry weight (MF BS), fresh weight (MF BU) and of dry leaves (FS) of Piper gaudichaudianum Kunth, in the four seasons of 2013 (two collections per season), in triplicate. Uppercase letters different indicate significant differences of yields between seasons and lowercase letters different indicate significant differences between yields in the same season, based on Tukey test, Mann-Whitney and test- $t, p<0.05$. 
$\mathrm{O}$ valor médio de rendimento na primavera para o OE de folhas frescas (MF BS) de Piper gaudichaudianum foi maior que nas demais estações, igual a 1,61 $\pm 0,09 \%$, coincidindo com o valor máximo de rendimento de OE em frutos (MOR BS) igual a 5,01\% (Figura 2). A presença de órgãos reprodutivos em três estações não alterou o rendimento do OE de folhas e apresentou crescente rendimento até o período máximo de floração e frutificação (inverno e primavera) (Figura 2). Estes resultados estão de acordo com dados da literatura, uma vez que Rodig e Poser (1990) verificaram um aumento de cerca de $20 \%$ no rendimento de OE de folhas de Piper gaudichaudianum coletadas em setembro no estado do Rio Grande do Sul. Este aumento nos teores de constituintes voláteis pode estar relacionado ao aumento do metabolismo da planta, decorrente do florescimento da espécie nesta época do ano. Conforme Gobbo-Neto e Lopes (2007), o rendimento dos OE é mais susceptível ao ciclo vegetativo que às variações climáticas, o que pode explicar a tendência no aumento do rendimento desse extrativo em inflorescências e frutos, do outono em direção à primavera. Em contrapartida, o rendimento do $\mathrm{OE}$ de folhas no verão foi o menor observado nesse estudo $(1,32 \pm 0,14 \%)$, período este em que a planta não se encontrava em estágio reprodutivo e/ ou a presença de inflorescências era pequena e estava em fase inicial de formação, não sendo possível sua coleta. Em um estudo desenvolvido por Costa et al. (2008), em um plantio da espécie Piper aduncum L. em Manaus (Brasil), o rendimento de OE foi menor também na fase vegetativa. $\mathrm{O}$ teor de umidade das folhas foi menor no verão $(73,59 \%$ ) (Tabela 2$)$, uma vez que se trata de um período mais seco, de temperaturas elevadas e baixa disponibilidade hídrica, o que, segundo Bernier et al. (1993), é um dos fatores que interfere no processo de floração.

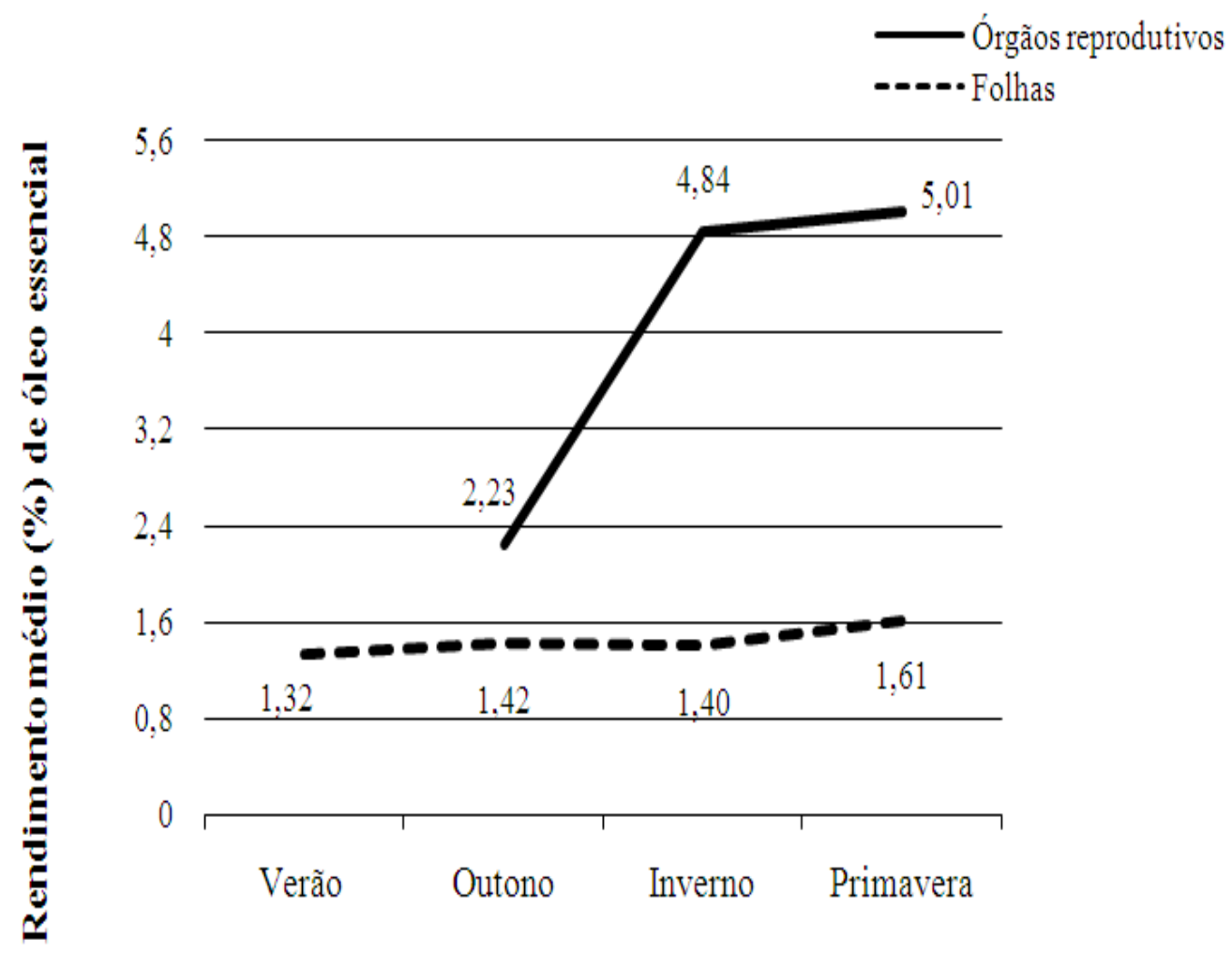

Estação do ano

FIGURA 2: Representação gráfica do rendimento sazonal do óleo essencial de folhas frescas e órgãos reprodutivos (outono e inverno: inflorescências; primavera: frutos), ambos à base seca (MF BS e MOR BS, respectivamente) de Piper gaudichaudianum.

FIGURE 2: Graphical representation of seasonal yield of the fresh leaves essential oils and reproductive organs (autumn and winter: inflorescences; spring: fruits), both based on dry weight (MF BS and MOR, respectively) of Piper gaudichaudianum. 
TABELA 2: Valores médios dos rendimentos (\%) de OE, considerando massa fresca à base seca (MF BS), massa fresca à base úmida (MF BU) e folhas secas (FS) de seis amostras por estação, no ano de 2013 (duas coletas por estação, em triplicata), bem como densidade do $\mathrm{OE}(\mathrm{g} / \mathrm{mL})$ de folhas MF BU e FS e teor de umidade (TU \%). EPM: ErroPadrão da Média.

TABLE 2: $\quad$ Average yields (\%) of EO considering fresh weight on dry basis (MF BS), on fresh basis (MF BU) and of dry leaves (FS) of six samples by season in 2013 (two collections, in triplicate), as well as OE density $(\mathrm{g} / \mathrm{mL})$ of leaves MF BS and moisture content (\% TU); SEM: Mean Standard Error.

\begin{tabular}{c|ccccc|c}
\hline \multirow{2}{*}{ Observações } & \multicolumn{4}{|c|}{ Estações } & \multirow{2}{*}{ Média \pm EPM } \\
\cline { 2 - 4 } & Verão & Outono & Inverno & Primavera & \\
\hline MF BS (m/m \%) & 1,32 & 1,42 & 1,40 & 1,61 & $1,44 \pm 0,060$ \\
MF BU (m/m \%) & 0,37 & 0,33 & 0,32 & 0,38 & $0,35 \pm 0,010$ \\
FS (m/m \%) & 1,24 & 1,22 & 1,25 & 1,53 & $1,31 \pm 0,060$ \\
Densidade do OE MF BU $(\mathrm{g} / \mathrm{mL})$ & 1,06 & 1,09 & 1,10 & 1,07 & $1,082 \pm 0,008$ \\
Densidade do OE FS $(\mathrm{g} / \mathrm{mL})$ & 1,07 & 1,11 & 1,09 & 1,03 & $1,086 \pm 0,010$ \\
TU (\%) & 73,59 & 76,75 & 75,77 & 76,03 & $75,90 \pm 0,680$ \\
\hline
\end{tabular}

Foram encontrados dados referentes ao rendimento do OE para folhas de três espécies do gênero Piper, entre elas Piper gaudichaudianum. Em Atalanta, Santa Catarina (Brasil), as folhas apresentaram teores entre 0,24 a $0,46 \%$ (SANTOS, 2009), inferiores aos relatados nesse trabalho. Adicionalmente, Rodig e Poser (1990) observaram que em uma população de Sapiranga - RS, o rendimento do OE de folhas foi de 0,30\% para o mês de maio. Já Morais et al. (2007) verificaram em Rondônia (Brasil) o teor de 0,01\%, para o OE de folhas.

O rendimento médio de OE (MF BS) foi comparado aos dados correspondentes de espécies do mesmo gênero. Desta maneira, os resultados aqui descritos são similares e intermediários aos teores de OE relatados para diferentes populações de Piper aduncum L. na região Amazônica (Brasil), que variaram de 1,2 a 3,3\% (MAIA et al., 1998) e para quatro populações do Distrito Federal (Brasil), que foram de 0,66 a 1,30\% (POTZERNHEIM et al., 2012). Entretanto, os rendimentos obtidos para o OE de folhas de Piper gaudichaudianum são superiores aos encontrados por Oliveira et al. (2013), em Montes Claros e Bocaiuva, para OE de plantas cultivadas e nativas em período de floração (0,3 a 0,7\%) e ao de Mesquita et al. (2005), obtido para Piper aduncum do Parque Estadual de Rio Doce (0,7\%), ambos no estado de Minas Gerais. Outras espécies apresentaram os rendimentos inferiores para o OE de folhas, como Piper malacophyllum (C. Presl) DC. (0,22 a 0,47\%) e Piper mikanianum (Kunth) Steud. (0,66 a 0,84\%) em Santa Catarina (SANTOS, 2009).

Salienta-se a importância da descrição dos fatores ambientais observados em cada local de coleta, pois pequenas modificações no ambiente podem ocasionar grandes alterações no rendimento do extrativo. Os indivíduos de Piper gaudichaudianum amostrados neste estudo estavam no sub-bosque da floresta, recebendo pouca luminosidade, sob um solo raso e rochoso. Segundo Maia et al. (1998), os indivíduos que ocorrem na Mata Atlântica são tolerantes à sombra, porém, apresentam crescimento bem mais rápido em locais sem dossel da floresta. Este aspecto é particularmente relevante quando se pretende fazer a reprodução da espécie para obtenção de seu OE para uso comercial. O estudo de Gobbo-Neto e Lopes (2007) indica que vários fatores exercem influência sobre o rendimento e composição química dos extrativos das plantas, entre eles a disponibilidade hídrica e nutricional, sazonalidade, altitude, poluição atmosférica, etc. Mesmo existindo um domínio genético, a expressão gênica pode sofrer modificações em consequência da interação de processos bioquímicos, evolutivos, fisiológicos e ecológicos.

Os rendimentos obtidos de FS foram comparados com os de MF BS, observando-se que não houve diferença significativa entre ambos para cada estação do ano. Essa informação é confirmada pela análise hierárquica de Cluster, realizada com os dados de todos os rendimentos, sendo obtido um dendrograma com dois grupos: A (MF BU) e B (MF BS e FS) (Figura 3). A correlação cofenética da análise resultou em 0,96 indicando que o agrupamento foi adequado, portanto, houve perda de massa por dessecação das folhas, porém, não ocorreu a diminuição do teor de OE. Uma vez que os rendimentos MF BS e FS foram 
superiores ao MF BU, o processo de secagem à temperatura ambiente pode ser recomendado como operação preliminar à extração.

Em espécies produtoras de $\mathrm{OE}$, estes podem ser produzidos e armazenados em estruturas secretoras internas (células parenquimáticas diferenciadas, bolsas lisígenas e canais oleíferos) ou externas (tricomas glandulares). Na família Piperaceae, os extrativos lipofílicos são encontrados em células parenquimáticas diferenciadas localizadas em tecidos internos de diferentes órgãos vegetais (BIASI; DESCHAMPS, 2009), o que fornece uma provável explicação para o fato do teor de OE não ter sido afetado pela secagem. Estudos morfoanatômicos realizados com folhas de Piper gaudichaudianum também indicam a presença de uma ou duas camadas subepidérmicas de natureza parenquimática (parênquima paliçádico) nas quais ocorrem os idioblastos oleíferos, localizados na face abaxial do limbo e no bordo (ALBIERO et al., 2005; FIGUEIREDO et al., 2008). Estes idioblastos secretores são células individualizadas de composição química distinta das células que as cercam; apresentam formato variável e são classificadas de acordo com as substâncias sintetizadas (CARDOSO, 2011). A presença de estruturas secretoras internas é o motivo pelo qual o OE contido nas mesmas possui uma volatilidade diferenciada em comparação ao presente em estruturas secretoras externas, que, dependendo do método de secagem, são intensamente afetados resultando em diminuição do teor de OE (VENSKUTONIS, 1997; HAMOROUNI SELLAMI et al., 2012).

É recomendável considerar também as demais vantagens que o processo de secagem oferece, como a maior estabilidade química do material vegetal seco, bem como a possibilidade de armazenamento antes do processo de extração (MARCHESE; FIGUEIRA, 2005). Figueiredo et al. (2004) relataram que folhas da espécie Piper hispidinervum C. DC. apresentam maior concentração de OE (em torno de 98\%) quando retira-se a umidade do material vegetal antes do processo de hidrodestilação. Deste modo, evita-se que ocorra a fermentação da biomassa e, consequentemente, perdas de rendimento e qualidade do $\mathrm{OE}$, sendo a secagem à sombra com ventilação forçada o método mais efetivo. No entanto, os resultados do presente estudo indicam que a secagem à sombra, em temperatura ambiente também é um método adequado. Além do mais, não é necessário processar o material vegetal imediatamente pós-colheita, o que seria particularmente problemático no caso da extração de grandes quantidades de matéria-prima, pois é possível fazer seu armazenamento após uma secagem apropriada.

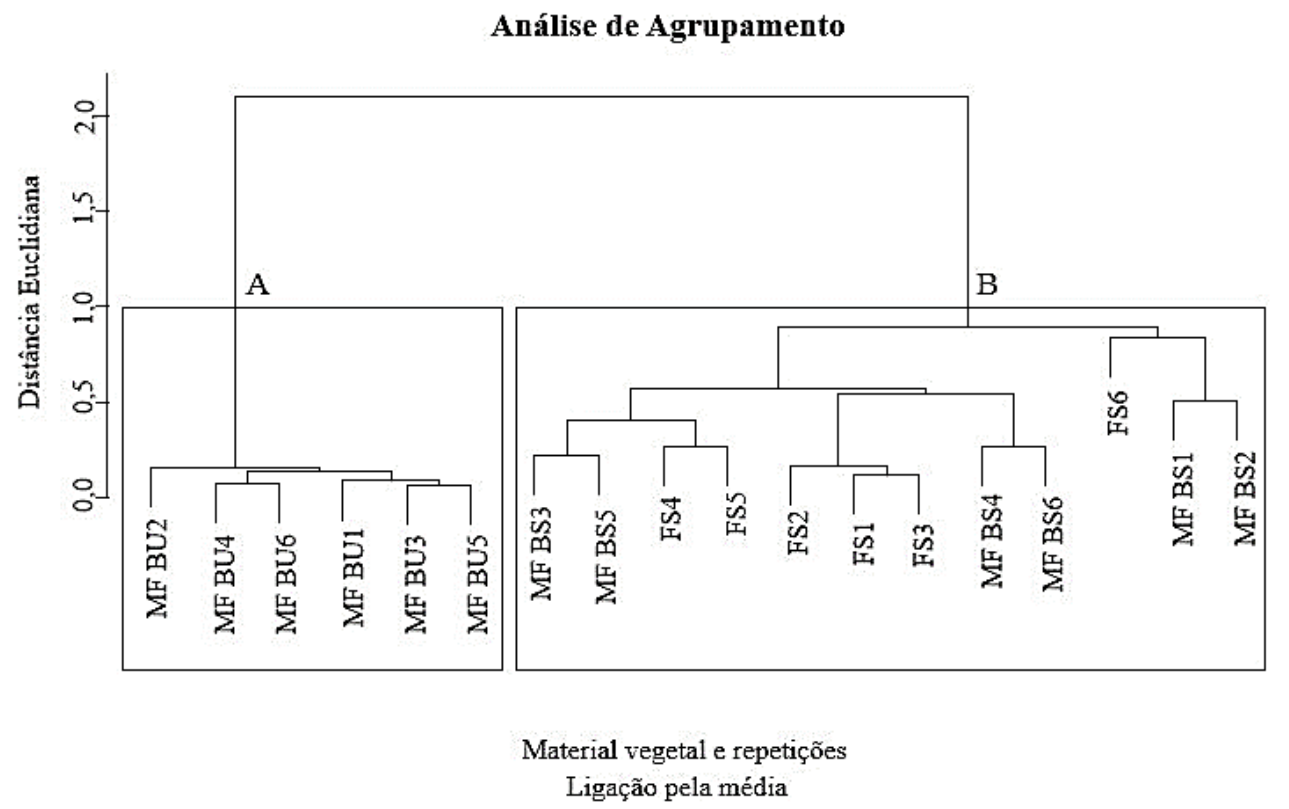

FIGURA 3:Dendrograma do rendimento (\%) de todas as amostras do óleo essencial de folhas de Piper gaudichaudianum, considerando MF BU, MF BS e FS (duas coletas em quatro estações no ano de 2013, com extrações em triplicata), através do método de ligação pela média e com a distância Euclidiana como medida de dissimilaridade.

FIGURE 3: Dendrogram of yield (\%) of all samples of the essential oil of Piper gaudichaudianum, considering MF BU, MF BS and FS (two collections in the four seasons of 2013, and extractions in triplicate) through the method of average linkage and the Euclidean distance as a measure of dissimilarity. 
Pesquisas envolvendo a composição química do OE de Piper gaudichaudianum, bem como a comparação entre diferentes métodos de secagem devem ser realizadas, a fim de se otimizar a obtenção deste extrativo. Não obstante, esta espécie pode ser considerada promissora, pois apresenta potencial para a produção de OE. Além disso, pode ser utilizada no enriquecimento de ambientes florestais perturbados ou com necessidade de recuperação, podendo tornar-se uma opção adicional de renda em locais cujo solo é impróprio para outras culturas.

\section{CONCLUSÕES}

Embora o melhor rendimento do OE de folhas de Piper gaudichaudianum tenha sido observado na primavera, período em que a planta se encontrava no estágio de maior maturação da frutificação (setembro a dezembro), esse extrativo não apresentou variação sazonal do rendimento para a população estudada no município de Santa Maria - RS. Portanto, a coleta de folhas para a obtenção desse extrativo pode ser realizada em qualquer época do ano. Além disso, o material vegetal pode ser submetido ao processo de secagem à temperatura ambiente, sem comprometer o rendimento do extrativo.

\section{AGRADECIMENTOS}

À Coordenação de Aperfeiçoamento de Pesquisa de Nível Superior (CAPES) e ao CNPq pelas bolsas e pelo financiamento concedido, bem como ao Engenheiro Florestal Maurício Figueira pela colaboração nas coletas do material vegetal.

\section{REFERÊNCIAS}

ALBIERO, A. L. M. et al. Morfo-anatomia do caule e da folha de Piper gaudichaudianum Kuntze (Piperaceae). Acta Farmacêutica Bonaerense, Buenos Aires, v. 24, n. 4, p. 550-554, 2005.

AMARAL, L. P. et al. Seasonal influence on the essential oil of Nectandra megapotamica (Spreng.) Mez. Brazilian Archives of Biology and Technology, Curitiba, v. 58, n. 1, p. 12-21, 2014.

BERNIER, G. et al. Physiological Signals That Induce Flowering. The Plant Cell, Waterbury, v. 5, n. 10, p. $1147-1155,1993$.

BIASI, L. A; DESCHAMPS, C. Plantas aromáticas: do cultivo à produção de óleo essencial. 1 ed. Curitiba/PR, Ed. Layer Studio Gráfico e Editora Ltda, 2009. 160 p.

BURT, S. Essential oils: their antibacterial properties and potential applications in foods - a review. International Journal of Food Microbiology, Amsterdam v. 94, n. 3, p. 223-253, 2004.

CARDOSO, P. R. Estruturas secretoras em plantas. 2011. 16 f. Estágio de Docência CAPES. Programa de Pós-Graduação em Biodiversidade Vegetal e Meio Ambiente, Instituto de Botânica, 2011.

COSTA, I. O. V. L. et al. Produção de Biomassa (Folhas e Caules) e Rendimento de Óleo Essencial de Piper aduncum L., em Função de Épocas de Colheita, nas Condições de Manaus - AM. In: JORNADA DE INICIAÇÃO CIENTIFICA DA EMBRAPA AMAZÔNIA OCIDENTAL, 3., 2008. Anais... [s. 1.: s. n], 2008. p. 89.

DI STASI, L. C.; HIRUMA-LIMA, C. A. Plantas medicinais na Amazônia e na Mata Atlântica. 2. ed. São Paulo: Ed. UNESP, 2002. 604 p.

FIEDLER, N. C.; SOARES, T. S.; SILVA, G. F. Produtos florestais não madeireiros: importância e manejo sustentável da floresta. Revista Ciências Exatas e Naturais, Guarapuava, v. 10, n. 2, 2008.

FIGUEIREDO, A. C. et al. Factors affecting secondary metabolite production in plants: volatile components and essential oils. Flavour and Fragrance Journal,Chinchester, v. 23, n. 4, p. 213-226, 2008.

FIGUEIREDO, F. J. C. et al. Secagem da biomassa aérea de pimenta longa sob condições de laboratório e de campo. Belém: Embrapa Amazônia Oriental, 2004. (Boletim de Pesquisa e Desenvolvimento, n. 32). GONÇALVES, E. G.; LORENZI, H. Morfologia vegetal: organografia e dicionário ilustrado de morfologia das plantas vasculares. 2. ed. São Paulo: Instituto Plantarum de Estudos da Flora, 2011. 512 p. GOBBO-NETO, L.; LOPES, N. P. Plantas medicinais: fatores de influência no conteúdo de metabólitos secundários. Química Nova, São Paulo, v. 30, n. 2, p. 374-381, 2007. 
GOTElli, N. J.; ELlisOn, A. M. Princípios de estatística em ecologia. Porto Alegre: Artmed, 2011. $527 \mathrm{p}$.

GOVINDASAMY, R.; ARUMUGAM, S.; SIMON, J. E. An assessment of the essential oil and aromatic plant industry with a focus on Africa. African Natural Plant Products, Washington,v. 2, n. 18, p. 289-321, 2013.

GUIMARÃES, E. F. et al. Piperaceae.In: JARDIM BOTÂNICO DO RIO DE JANEIRO. Lista de Espécies da Flora do Brasil. 2014. Disponível em: <http://floradobrasil.jbrj.gov.br/2012/FB012738>. Acesso em: 18 maio 2014.

GUimarÃES, E. F.; VALENTE, M. C. Piperáceas - Piper. Flora Ilustrada Catarinense. Itajaí: [S. L.], 2001. 103 p.

HAMROUNI SELLAMI, I. et al. Drying sage (Salvia officinalis L.) plants and its effects on content, chemical composition, and radical scavenging activity of the essential oil. Food and Brioprocess Technology, Berlim, v. 5, n. 8, p. 2978-2989, 2012.

HELDWEIN, A. B.; BURIOL, G. A.; STRECK, N. A. O clima de Santa Maria. Ciência \& Ambiente, Santa Maria, n. 38, p. 43-58, 2009.

IBGE. Manual técnico da vegetação brasileira. Rio de Janeiro: IBGE, 1992. 92 p.

LAGO, J. H. G. et al. Benzoic acid derivatives from Piper species and their fungitoxic activity against Cladosporium cladosporioides and C. shaerospermum. Journal of Natural Products, Cincinnati, v. 67, n. 11. p. 1783-1788, 2004.

LIMA, H. R. P.; KAPLAN, M. A. C.; CRUZ, A. V. M. Influência dos fatores abióticos na produção e variabilidade de terpenóides em plantas. Floresta e Ambiente, Seropédica, v. 10, n. 2, p. 71-77, 2003.

MAIA, J. G. S. et al. Constituents of the essential oil of Piper aduncum L. growing wild in the Amazon region. Flavour and Fragrance Journal, Chinchester, v. 13, n. 4, p. 269-272, 1998.

MACHADO, F. S. Manejo de produtos florestais não madeireiros: um manual com sugestões para o manejo participativo em comunidades da Amazônia. Rio Branco: PESACRE; CIFOR, 2008.

MACIEL FILHO, C. L. Carta geotécnica de Santa Maria. Santa Maria: Imprensa Universitária, 1990. $24 \mathrm{p}$.

MARCHESE, J. A.; FIGUEIRA, G. M. O uso de tecnologias pré e pós-colheita e boas práticas agrícolas na produção de plantas medicinais e aromáticas. Revista Brasileira de Plantas Medicinais, Paulinia, v. 7 , n. 3, p. 86-96, 2005.

MESQUITA, J. M. O. et al. Estudo comparativo dos óleos voláteis de algumas espécies de Piperaceae. Revista Brasileira de Farmacognosia, Curitiba, v. 15, p. 6-12, 2005.

MIKICH, S. B. et al. Attraction of the fruit-eating bat Carollia perspicillata to Piper gaudichaudianum essential oil. Journal of Chemical Ecology, New York, v. 29, n. 10, p. 2380-2383, 2003.

MOREIRA, D. L. et al. Estudos fitoquímico e farmacológico de Piper gaudichaudianum Kunth (Piperaceae).

Revista Brasileira de Farmácia, Rio de Janeiro, v. 82, n. 1/2, p. 29-32, 2001.

MORAIS, S. M. et al. Chemical composition and larvicidal activity of essential oils from Piper species.

Biochemical Systematics and Ecology, Oxford, v. 35, n. 10, p. 670-675, 2007.

OLIVEIRA, G. L. et al. Growth study and essential oil analysis of Piper aduncum L. from two sites of Cerrado biome of Minas Gerais State, Brazil. Revista Brasileira de Farmacognosia, Curitiba, v. 23, p. 743-753, 2013.

OLIVEIRA-FILHO, A. T. Classificação das Fitofisionomias da América do Sul-Cisandina Tropical e Subtropical: proposta de um novo sistema - prático e flexível - ou uma injeção a mais de caos? Rodriguésia, Rio de Janeiro, v. 2, n. 60, p. 237-258, 2009.

OKSANEN, J. et al. Community Ecology Package. [s. 1.: s. n.], 2013.

POTZERNHEIM, M. C. L. et al. Chemical characterization of essential oil constituents of four populations of Piper aduncum L. from Distrito Federal, Brazil. Biochemical Systematics and Ecology, Oxford, v. 42, p. 25-31, 2012

RODIG, L. R.; POSER, G. L. V. Constituintes químicos de espécies da família Piperaceae. Acta Biologica Leopoldensia, São Leopoldo, p. 57-64, 1990.

ROHLF, F. J. Adaptative hierarquical clustering schemes. Systematic Zoology, Oxford, v. 19, n. 1, p. 58$82,1970$. 
SANTOS, A. J. et al. Produtos não madeireiros: conceituação, classificação, valoração e mercados. Revista Floresta, Curitiba, v. 33, n. 2, p. 215-224, 2003.

SANTOS, T. G. Composição química e atividade antimicrobiana dos óleos essenciais de três espécies do gênero Piper e de Baccharis semiserrata DC. 2009. 117 f. Dissertação (Mestrado em Química) Universidade Regional de Blumenau, Blumenau, 2009.

SARTOR, R. B. Modelagem, simulação e otimização de unidade industrial de extração de óleos essenciais por arraste a vapor. 2009. 75 f. Dissertação (Mestrado em Engenharia Química) - Universidade Federal do Rio Grande do Sul, Porto Alegre, 2009.

SILVA, R. S. et al. Óleo essencial de limão no ensino da cromatografia em camada delgada. Química Nova, São Paulo, v. 32, n. 8, p. 2234-2237, 2009.

SOIDROU, S. H. et al. Fungicidal activity of four essential oils from Piper capense, Piper borbonense and Vetiveria zizanoides growing in Comoros against fungi decay wood. The Journal of Essential Oil Research, Carol Stream, v. 25, n. 3, p. 216-223, 2013.

SPEZIALI, M. G. De aromas e perfumes, o mercado da indústria do "cheiro". Química Nova, São Paulo, v. 35, n. 4, p. 861-864, 2012.

STRECK, E. V. et al. Solos do Rio Grande do Sul. 2. ed. Porto Alegre: EMATER, 2008. 222 p.

VENSKUTONIS, P. R. Effect of drying on the volatile constituents of thyme (Thymus vulgaris L.) and sage (Salvia oficinalis L.). Food Chemistry, London, v. 59, n. 2, p. 219-227, 1997. 